\title{
Continuity effects with alternately sounding tones under dichotic presentation'
}

\author{
LLOYD F. ELFNER AND JERRY L. HOMICK \\ KENT STATE UNIVERSITY
}

An experiment is reported in which 10 Ss possessing normal hearing were required to make discriminations of continuity or interruption in a longer less intense signal (Tone A) which alternated in time with a shorter more intense signal (Tone $B)$. The signals were presented dichotically with Tone A at the right ear. Three Tone B frequencies of 300, 1000, and 4000 cps and five Tone A frequencies somewhat near each of the Tone $B$ frequencies were employed. The results demonstrated that as Tone A was nearer to Tone B in frequency, continuity thresholds in Tone A occurred at longer durations of Tone B. The results are discussed in terms of a central neural model.

Continuity effects in alternately sounded signals were first demonstrated by Thurlow (1957). He found that under certain conditions, when two tones near in frequency are alternately sounded, the tone of lower intensity and longer duration is perceived as continuous. In investigating various aspects of this phenomenon it has been demonstrated that continuity effects can be achieved when white noise is used as the longer, less intense signal and tone as the alternating signal (Elfner \& Caskey, 1965). This study also demonstrated that the duration of the white noise and the frequency of the tone produce significant changes in continuity thresholds under both monaural and dichotic presentation. However, a subsequent study (Elfner \& Homick, 1966) failed to find a significant change in continuity thresholds as a function of the frequency of the interpolated tone under dichotic presentation. In the above studies, continuity thresholds in the white noise were determined by varying the duration of the interpolated tone. An alternate method of studying the continuity effect is that of changing the intensity relationships between the alternating signals. In a continuity study employing two monaurally presented tones, Thurlow and Elfner (1959) found that a longer, less intense tone (Tone A) can be made to sound continuous by increasing the intensity of the other shorter tone (Tone B). This study also demonstrated that the intensity difference between Tone A and Tone B necessary to produce continuity is less when the two tones are near in frequency.

The main purpose of the present study was to investigate continuity effects in alternately sounded tonal signals under dichotic presentation.

\section{Subjects}

\section{METHOD}

Ten Ss from undergraduate psychology courses at Kent State University were employed. All Ss demon- strated normal hearing and an ability to listen for continuity in a background tone in the presence of an alternately sounded tonal burst.

\section{Apparatus}

Tones $\mathrm{A}$ and $\mathrm{B}$ were produced by two separate audio-oscillators (Hewlett-Packard, Models 241A and 204B). Each tone was led from its oscillator into one channel of an electronic switch (Grason-Stadler, Model 829D) and interval timer (Grason-Stadler, Model 471-1). The switch and timer produced an alternation of the two signals in time. The tones were then led separately through a timer (Hunter, Model 100-C) to decade attenuators (Hewlett-Packard, Model 305D) and then to a transformer (Koss, Model T-1). From here the signal went to earphones (Koss, Model SP-3) located in an audiometric test chamber (IAC, Model 1201A) where the $S$ listened. The signals were calibrated and monitored on an oscilloscope.

\section{Procedure}

The data were gathered in two sessions for each $\mathrm{S}$. Initially, absolute thresholds were determined for each of the frequencies employed. Five Tone A frequencies were used for each of the three Tone $B$ frequencies. The Tone A signals used with the $300 \mathrm{cps}$ Tone B were $200,250,400,500$, and $700 \mathrm{cps}$. The Tone A signals used with the 1000 cps Tone B were 600, $800,1500,2000$, and 2500 cps. The Tone A signals used with the $4000 \mathrm{cps}$ Tone B were 2000,3000, 4500,5000 , and $6000 \mathrm{cps}$. A rise-decay time of $1 \mathrm{msec}$. was used for all signals. Absolute thresholds for the above frequencies were obtained by the Method of Limits with six alternately ascending and descending threshold crossings. Tones A and B were presented at 30 and $45 \mathrm{~dB}$ sensation level, respectively, in order to minimize the probability of cross head conduction. Tone A was presented at a duration of $250 \mathrm{msec}$. for all conditions. Thresholds of interruption in Tone A were first obtained by varying the duration of a silent interval in the Tone A pulses (that is with Tone $B$ absent). Continuity thresholds in Tone A were then determined by varying the duration of Tone $B$. The threshold of continuity, or interruption, was defined as the point at which the perception of Tone A changed from continuous to interrupted or vice-versa. All continuity thresholds were determined by the Method of Limits and four alternately ascending and descending trials were used. The order of presentation was random. $S$ reported verbally via an intercom whether Tone A sounded continuous or interrupted. 


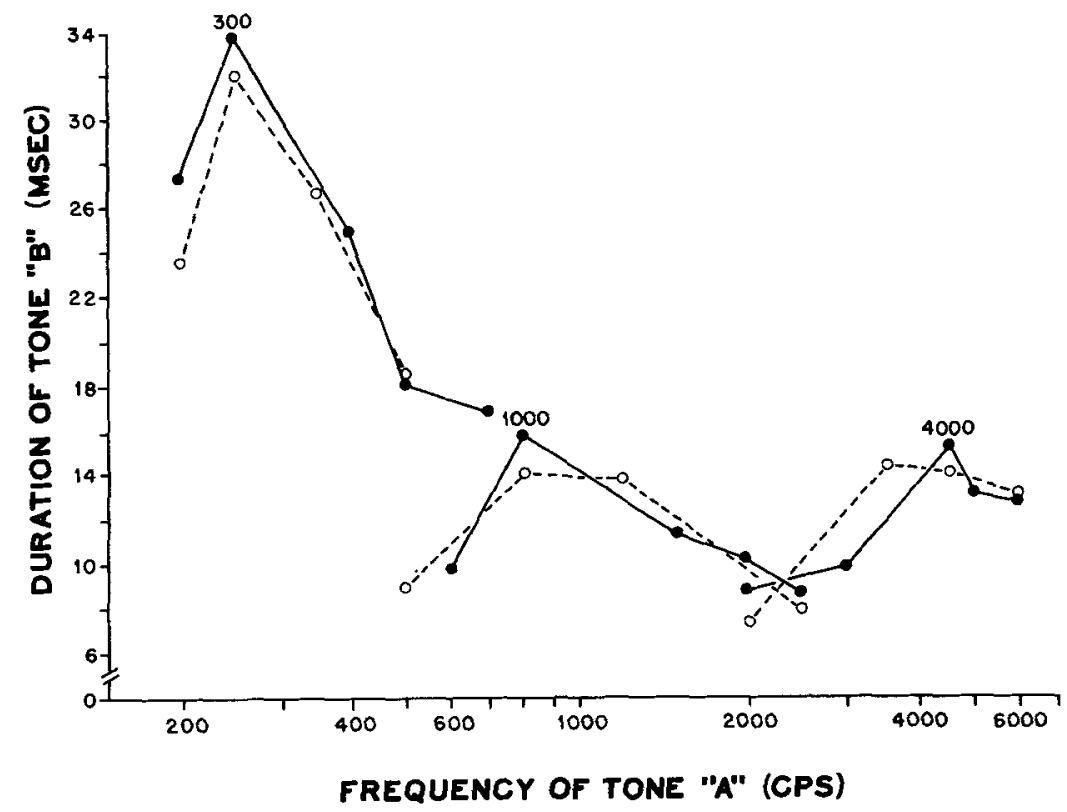

The results of the study are shown by the solid lines in Fig. 1.

The duration of Tone B necessary to produce continuity in Tone $A$ is plotted as a function of the frequency of Tone A. A separate plot is made for each of the Tone $B$ frequencies. It can be seen that as Tone $A$ is nearer in frequency to Tone $B$ thresholds for continuity in Tone A occur at longer durations of Tone B; that is, continuity occurs at longer interruptions in Tone A. The same general effect is seen at each of the three Tone B frequencies. Analyses of variance for repeated measures (Winer, 1962, p. 369) were performed on the obtained continuity thresholds for each of the Tone $B$ frequencies. The results summarized in Table 1, show that in all cases the frequency of Tone $A$ is a significant factor in the continuity effect $(p<.01)$.

The broken lines in Fig. 1 represent the results of a replication of the study. Nine Ss who had not previously served in continuity studies were employed. The four Tone A signals used with the $300 \mathrm{cps}$ Tone B were $200,250,350$ and 500 cps. The Tone A signals used with the 1000 cps Tone B were $500,800,1200$, and $2500 \mathrm{cps}$. The Tone A signals used with the 4000 cps Tone B were $2000,3500,4500$, and $5000 \mathrm{cps}$. In all other aspects the two studies were identical. As can be seen the results of the two studies are quite similar.

The mean thresholds for interruption in Tone $A$ (Tone B absent) were as follows: (1) for $400 \mathrm{cps}, 2.5$ msec., (2) for $1500 \mathrm{cps}, 2.7$ msec., (3) for $4500 \mathrm{cps}$, $3.7 \mathrm{msec}$. No analyses were made to compare continuity versus interruption thresholds since at all three Tone B frequencies, thresholds for continuity were considerably larger.
Fig. 1. Duration of the shorter, more intense signal (Tone $B$ ) at which the threshold for continuity is found in the longer, less intense signal (Tone A). Results are given for three Tone $B$ settings, 300, 1000 , and $4000 \mathrm{cps}$. The area below each curve represents the region of continuity. The broken curves represents the results of a replication.

\section{Discussion}

It is apparent from the preceding results that under conditions of dichotic presentation a shorter, more intense tone, presented in the interruptions of another longer, less intense tone can cause that other tone to sound continuous. It is also evident that the frequency of the interpolated signal has a significant effect on the threshold of continuity. The effect appears to be greatest when the two signals are not too far removed in frequency. This result is similar to that obtained by Elfner and Thurlow (1959) under monaural presentation. Although limited in nature, there is some evidence to suggest that masking may

Table 1. Repeated measures analyses of variance of the effects of the frequency of Tone $A$ on continuity thresholds for each of the Tone $B$ frequencies employed.

\begin{tabular}{|c|c|c|c|c|c|}
\hline \multicolumn{6}{|c|}{300 cps Tone B } \\
\hline Source & SS & df & MS & $\mathrm{F}$ & p \\
\hline Subjeets (Ss) & 1784.83 & 9 & 198.31 & & \\
\hline Frequency $(A)$ & 1928.76 & 4 & 482.19 & 15.74 & $<.01$ \\
\hline $\mathrm{A} \times \mathrm{Ss}_{\mathbf{s}}$ & 1102.75 & 36 & 30.63 & & \\
\hline Totol & 4816.34 & 49 & & & \\
\hline \multicolumn{6}{|c|}{1000 cps Tone B } \\
\hline Subjects (Ss) & 228.42 & 9 & 25.38 & & \\
\hline Frequency $(A)$ & 319.61 & 4 & 79.90 & 9.88 & $<.01$ \\
\hline $\mathrm{A} \times \mathrm{Ss}_{\mathbf{s}}$ & 290.95 & 36 & 8.08 & & \\
\hline Total & 838.98 & 49 & & & \\
\hline \multicolumn{6}{|c|}{$4000 \mathrm{cps}$ Tone 8} \\
\hline Subjects $\left(S_{s}\right)$ & 772.18 & 9 & 85.80 & & \\
\hline $\begin{array}{l}\text { Frequency }(A) \\
A \times S_{s}\end{array}$ & $\begin{array}{l}263.37 \\
264.71\end{array}$ & $\begin{array}{r}4 \\
36\end{array}$ & $\begin{array}{r}65.84 \\
7.35\end{array}$ & 8.96 & $<.01$ \\
\hline Total & 1300.26 & 49 & & & \\
\hline
\end{tabular}


be involved in the continuity effect. Ingham (1959) has examined the differential masking effect of a tone upon another tone in the opposite ear when the frequency separation of the tones was varied. He found that the masking effect decreased as the frequency separation of the tones increased. However, Ingham used a simultaneous masking technique whereas the continuity effect would necessarily entail forward and backward masking. Because of the low sensation levels employed (30 dB masking signal) Ingham suggests a central neural mechanism must be operating in cross-ear masking effects. Likewise, Thurlow and Elfner (1959) suggest a central mechanism to explain continuity effects. The results of the present study provide further support for such an explanation.

Some neurophysiological evidence for a model of binaural interaction that could explain the above effects has been reported by Rupert et al (1966). They studied neural response patterns of medial superior-olivary units (MSO) to auditory stimuli under both monaural and binaural stimulation. Their model assumes that "corresponding portions of the basilinear membrane of each ear are functionally represented at the same site within the MSO in as much as results obtained with tonal stimuli suggest a convergence of fibers upon MSO cells that produce interactive effects best when the frequencies to each ear are the same." The maximum enhancement of the continuity effect which occurs when the alternating signals are close in frequency, and the similarity of the frequency effect on continuity under monaural and dichotic presentation appear consistent with the above model.

The effect of frequency of the interpolated signal on continuity in a white noise signal is not clear. The typical result is to find significant frequency effects only under monaural listening procedures. Most of the differential effect of frequency on continuity in white noise seems to be a function of frequencies in the neighborhood of 1000 cps (Elfner \& Homick, 1966). The results of preliminary work employing restricted bands of noise alternating with tonal burst indicate that continuity in a tonal signal is enhanced when its frequency is centered in the noise band with which it alternates as opposed to being removed from that of the noise band. However, at this time, no simple explanation of the effect of frequency of a tonal burst on continuity of an alternating background noise is apparent.

\section{References}

Elfner, L. F., \& Caskey, W. E. Continuity effects with alternately sounded noise and tone signals as a function of manner of presentation, J. Acoust. Soc. Amer., 1965, 38, 543-547.

Elfner, I. F., \& Homick, J. L. Some factors affecting the perception of continuity in alternately sounded tone and noise signals. J. Acoust. Soc. Amer., 1966, in print.

Ingham, J. G. Variations in cross masking with frequency. $J$. exp. Psychol., 1959, 58, 199-205.

Thurlow, W. R. An auditory figure-ground effect. Amer. J. Psychol. 1957, 70, 653-654.

Rupert, A., Moushegian, G., \& Whitcomb, M. A. Superior-olivary response patterns to monaural and binaural clicks. J. Acoust. Soc. Amer., 1966, 39, 1069-1076.

Winer, B. J. Statistical principles in experimental design. New York: McGraw-Hill, 1962.

\section{Note}

1. This research was supported by the National Science Foundation under Grant GB 3475 .

(Accepted for publication October 26, 1966.)

\section{Erratum}

STONE, H., \& OLIVER, SHIRLEY M. Beidler's theory and human taste stimulation. Percept. \& Psychophys., 1966, 1, 358-360.-The article referred to on page 359 , column 2 , paragraph 3 , fourth line from the end was omitted from the bibliography. The reference was: Amerine, M. A., Pangborn, R. M., and Roessler, E. B. Principles of sensory evaluation of food. New York: Academic Press, Inc., 1965 , pp. 63-64. 